\title{
69. A Note on Compact Space
}

\author{
By Kiyoshi IséKI
}

(Comm. by K. KunUGI, M.J.A., May 15, 1957)

In this short Note, we shall give a remark for my results $[2,4]$. Following E. Hewitt, we shall define a pseudo-compact space as follows. A completely regular $T_{2}$-space $S$ is called pseudo-compact, if every continuous function on $S$ is bounded. In his paper [1], R. Doss has proved that if, in a uniform $T_{2}$-space $S$, every continuous function reaches its upper bound, then $S$ is precompact. This is a generalisation of a result of M. Fréchet. Therefore, this implies the following result. Any pseudo-compact uniform space is precompact. Hence, any pseudo-compact compelete uniform space is compact. It is well known that every uniform space with Lebesgue property is paracompact complete (see [3] or [5]). Hence, by Theorem 2 in [4], we have the following propositions:

Proposition 1. Every pseudo-compact complete uniform space is compact.

Proposition 2. The following statements of a uniform space $S$ with Lebesgue property are equivalent:

1) $S$ is compact.

2) $S$ is pseudo-compact.

3) $S$ is countably compact.

Proposition 2 was obtained in [2] by an other method.

\section{References}

[1] R. Doss: On continuous functions in uniform spaces, Ann. Math., 48, 843-844 (1947).

[2] K. Iséki: On the property of Lebesgue in uniform spaces. III, Proc. Japan Acad., 31, 441-442 (1955).

[3] K. Iséki: On the Lebesgue property in uniform spaces, Publ. Math., Debrecen, 4, 239-241 (1956).

[4] K. Iséki: A remark on countably compact normal space, Proc. Japan Acad., 33, 131-133 (1.957).

[5] S. Kasahara: On the Lebesgue property in uniform spaces, Math. Japonicae, 3, 127-132 (1955). 